\title{
Hyperdynamic Microtubules, Cognitive Deficits, and Pathology Are Improved in Tau Transgenic Mice with Low Doses of the Microtubule-Stabilizing Agent BMS-241027
}

\author{
Donna M. Barten, ${ }^{1 *}$ Patrizia Fanara, ${ }^{2 *}$ Cathy Andorfer, ${ }^{3}$ Nina Hoque, ${ }^{1}$ P. Y. Anne Wong, ${ }^{2}$ Kristofor H. Husted, ${ }^{2}$ \\ Gregory W. Cadelina, ${ }^{1}$ Lynn B. DeCarr, ${ }^{1}$ Ling Yang, ${ }^{1}$ Victoria Liu, ${ }^{2}$ Chancy Fessler, ${ }^{2}$ Joan Protassio,, 2 Timothy Riff, ${ }^{2}$ \\ Holly Turner, ${ }^{2}$ Christopher G. Janus, ${ }^{3}$ Sethu Sankaranarayanan, ${ }^{1}$ Craig Polson, ${ }^{1}$ Jere E. Meredith, ${ }^{1}$ Gemma Gray, ${ }^{3}$ \\ Amanda Hanna, ${ }^{3}$ Richard E. Olson, ${ }^{1}$ Soong-Hoon Kim, ${ }^{4}$ Gregory D. Vite, ${ }^{4}$ Francis Y. Lee, ${ }^{4}$ and Charles F. Albright ${ }^{1}$ \\ ${ }^{1}$ Neuroscience Drug Discovery, Bristol-Myers Squibb, Wallingford, Connecticut 06492, ${ }^{2}$ KineMed, Inc., Emeryville, California $94608,{ }^{3}$ Mayo Clinic College \\ of Medicine, Jacksonville, Florida 32224, and ${ }^{4}$ Oncology Drug Discovery, Bristol-Myers Squibb, Princeton, New Jersey 08543
}

Tau is a microtubule (MT)-stabilizing protein that is altered in Alzheimer's disease (AD) and other tauopathies. It is hypothesized that the hyperphosphorylated, conformationally altered, and multimeric forms of tau lead to a disruption of MT stability; however, direct evidence is lacking in vivo. In this study, an in vivo stable isotope-mass spectrometric technique was used to measure the turnover, or dynamicity, of MTs in brains of living animals. We demonstrated an age-dependent increase in MT dynamics in two different tau transgenic mouse models, 3xTg and $\mathrm{rTg} 4510$. MT hyperdynamicity was dependent on tau expression, since a reduction of transgene expression with doxycycline reversed the MT changes. Treatment of rTg4510 mice with the epothilone, BMS-241027, also restored MT dynamics to baseline levels. In addition, MT stabilization with BMS-241027 had beneficial effects on Morris water maze deficits, tau pathology, and neurodegeneration. Interestingly, pathological and functional benefits of BMS-241027 were observed at doses that only partially reversed MT hyperdynamicity. Together, these data suggest that tau-mediated loss of MT stability may contribute to disease progression and that very low doses of BMS-241027 may be useful in the treatment of AD and other tauopathies.

\section{Introduction}

Tauopathies are neurodegenerative diseases in which neurons accumulate abnormal forms of tau, including hyperphosphorylated, conformationally altered, mislocalized, fragmented, oligomeric, and fibrillar forms (Lace et al., 2007; Brunden et al., 2009; Ittner et al., 2011; Morris et al., 2011). Alzheimer's disease (AD) is the most common tauopathy, which has the additional pathological hallmark of extracellular plaques composed of $\beta$-amyloid $(\mathrm{A} \beta)$ peptides. In $\mathrm{AD}$, abnormalities in tau are believed to be downstream of $\mathrm{A} \beta$ oligomers. In some tauopathies, particularly

Received Jan. 13, 2012; revised March 26, 2012; accepted March 30, 2012.

Author contributions: D.M.B., P.F., C.A., S.S., J.E.M., F.Y.L., G.D.V., and C.F.A. designed research;D.M.B., P.F., C.A., N.H., P.Y.A.W., K.H.H., G.W.C., L.B.D., L.Y., V.L., C.F., J.P., T.R., H.T., C.G.J., C.P., G.G., A.H., S.-H.K., and R.E.O. performed research; D.M.B., P.F., C.A., and L.B.D. analyzed data; D.M.B., P.F., C.G.J., S.S., C.P., J.E.M., R.E.O., and C.F.A. wrote the paper.

All funding for the research was provided by Bristol-Myers Squibb. We would like to acknowledge the contributions of David Stock, Antara Majumdar, and Bradley Snyder toward statistical analyses. Karen Phillips, Linda Rousseau, and Virginia Phillips assisted with tissue sectioning, and Dennis Dickson provided silver staining support. Jada Lewis provided some of the mice used in the studies. We also thank John Corradi, Michael Ahlijanian, and Paul Wes for helpful editorial comments.

${ }^{*}$ D.M.B. and P.F. contributed equally to this publication.

D.M.B., N.H., G.W.C., L.B.D., L.Y., S.S., C.P., J.E.M., F.Y.L, S.-H.K., G.D.V., R.E.O., and C.F.A. are employees of Bristol-Myers Squibb. P.F., P.Y.A.W., K.H.H., V.L., C.F., J.P., T.R., and H.T. are employees of KineMed, Inc.

Correspondence should be addressed to Dr. Donna M. Barten, Neuroscience Drug Discovery, Bristol-Myers Squibb, 5 Research Parkway, Wallingford, cT 06492. E-mail: donna.barten@bms.com.

C. Andorfer's present address: Comprehensive Cancer Center, Mayo Clinic, 4500 San Pablo Road, Jacksonville, FL 32224.

DOI:10.1523/JNEUROSCI.0188-12.2012

Copyright $\odot 2012$ the authors $\quad 0270-6474 / 12 / 327137-09 \$ 15.00 / 0$ frontotemporal dementia with parkinsonism type-17 (FTDP17), tau mutations, such as P301L, can initiate disease (Ittner et al., 2011).

Tau binds to and modifies microtubule (MT) dynamics (Drechsel et al., 1992; Weissmann et al., 2009). MTs, made up of tubulin dimers, are critical to bidirectional transport of cargo to the synapse. Disease-associated forms of tau are hypothesized to interfere with the normal MT-stabilizing function of tau. Hyperphosphorylated tau isolated from AD brain binds less well to MTs (Lu and Wood, 1993), and abnormal tau may also sequester normal tau (Alonso et al., 1996; Mocanu et al., 2008). FTDP-17 tau mutations also alter stabilization of MTs or interfere with the docking of motor proteins (Feinstein and Wilson, 2005). If alterations in MT transport result from tau abnormalities, then an MT-stabilizing agent that restores MT function could be therapeutic (Brunden et al., 2009).

MT stabilizers, such as epothilones and taxanes, are used for the treatment of cancer. These molecules are cytotoxic to dividing cells by interfering with MT-dependent chromosome separation. On the other hand, MT stabilizers are neuroprotective for A $\beta$ mediated toxicity (Michaelis et al., 2002; Silva et al., 2011), enhance neurite outgrowth (Sengottuvel et al., 2011), and reduce deficits induced by tau-P301L transfection (Shemesh and Spira, 2011) in cultured neurons. In vivo, paclitaxel (Taxol), which does not have good brain penetration, improves axonal transport rate, MT numbers, and motor function in a spinal cord tauopathy model (Zhang et al., 2005). In spinal cord injury and optic nerve 
crush models, local administration of paclitaxel improves axonal regeneration and motor function (Hellal et al., 2011; Sengottuvel et al., 2011). In addition, NAP, a peptide with possible indirect MT-stabilizing activity (Yenjerla et al., 2010), has pathological and behavioral benefits in several tauopathy models (Gozes et al., 2009). NAP is in Phase II clinical trials for tauopathy.

BMS-241027, also called epothilone D, KOS862, dEpoB, and CRND66, is a brain-penetrant MT stabilizer (Kolman, 2004). Phase II oncology trials with BMS-241027 at maximum tolerated doses were discontinued due to poor activity and neurological side effects (Beer et al., 2007). At low doses in a tauopathy model, BMS-241027 reduces axonal dystrophy, increases MT numbers, reduces pathology, and improves axonal transport and cognitive performance (Brunden et al., 2010; Zhang et al., 2012).

Here we provide data supporting the hypothesis that abnormalities in tau lead to alterations in MT turnover in vivo. MT hyperdynamicity was reduced following alterations of tau transgene expression or with BMS-241027 treatment in a tauopathy model. MT stabilization by BMS-241027 also reduced pathology and cognitive decline. Phase I clinical trials with low doses of BMS-241027 in AD patients are being initiated.

\section{Materials and Methods}

Animal handling. Mice were handled strictly according either to BristolMyers Squibb (BMS), Mayo Clinic, or KineMed Animal Care and Use Committee guidelines. Mice of only a single sex were used for most studies and is indicated in figure legends. The $n$ value for each study is reported either in the figure, as individual data per mouse, or in the legend. 3xTg mice (Oddo et al., 2003) were bred at BMS and were obtained congenic in the C57BL/6J background from Mark Mattson (National Institute on Aging, Baltimore, MD). rTg4510 mice (Santacruz et al., 2005) were bred at the Mayo Clinic or BMS. Both 3xTg and rTg4510 lines express the 4R0N P301L mutant form of human tau (tau-P301L). rTg4510 mice have two transgenes: a tetracycline-controlled transcriptional activator $(t T A)$ driven by the $\mathrm{Ca}^{2+} /$ calmodulin-dependent protein kinase II $\alpha$ promoter and a tau-P301L transgene driven by a tetracycline operon-responsive element (TRE). Control lines either contain the transcriptional activator alone (tTA Alone), the tau transgene alone (Tau Alone), or neither transgene (Dble Neg). Mice were housed with a 6:00 A.M. to 6:00 P.M. light/dark cycle and allowed free access to food and water. For doxycycline treatment, mice were fed chow containing 200 ppm doxycycline (Barten et al., 2011). BMS-241027 was dosed intraperitoneally once weekly, in $10 \%$ ethanol, $90 \%$ water, or in $8.5 \%$ polyethylene glycol, average molecular weight of $400,1 \% \mathrm{D}-\alpha$-tocopheryl polyethylene glycol 1000 succinate, $0.5 \%$ ethanol, $90 \%$ water. A $10 \times$ stock solution was made in the organic phase and diluted in water just before dosing. For pathological analyses, mice were killed, and the brain was removed and fixed in $4 \%$ paraformaldehyde for paraffin embedding and sectioning.

${ }^{2} \mathrm{H}_{2} \mathrm{O}$ labeling and MT dynamics assay. This assay was performed as described previously (Fanara et al., 2010) with minor modifications. In brief, mice received an intraperitoneal bolus of $30-35 \mathrm{ml} / \mathrm{kg}^{2} \mathrm{H}_{2} \mathrm{O}(99.9$ mol\%) in saline and were then maintained on $8 \%{ }^{2} \mathrm{H}_{2} \mathrm{O}$ in drinking water for $24 \mathrm{~h}$ before being killed. This labeling protocol results in $4-5 \%$ body water deuterium enrichment. Mice were anesthetized by inhalation of $2.5 \%$ vaporized isoflurane in $2 \mathrm{~L} / \mathrm{min}_{2}$, followed by cardiac puncture to collect plasma for body water enrichment analysis and removal of the brain. A $2 \mathrm{~mm}$ slice of cortex was dissected above the ventral hippocampus. In one study, a $2 \mathrm{~mm}$ slice of cerebellum was collected as well. The tissue was placed in $2 \mathrm{ml}$ of microtubule-stabilizing buffer (MSB) at room temperature. MSB contained $46 \mathrm{~mm}$ 1,4piperazinediethanesulfonic acid (PIPES) buffer, $\mathrm{pH}$ 6.8, $0.6 \mathrm{~mm}$ magnesium chloride $\left(\mathrm{MgCl}_{2}\right), 1 \mathrm{~mm}$ EGTA, $10 \%$ glycerol, $0.2 \mathrm{M}$ sucrose, $1 \mathrm{~mm}$ GTP, and protease and phosphatase inhibitors. The tissue was homogenized with a Polytron, taking care not to let the samples overheat. The homogenate was centrifuged at $8500 \times g$ for $10 \mathrm{~min}$ at $25^{\circ} \mathrm{C}$. The resulting supernatant was centrifuged either at $245,000 \times g$ for $19 \mathrm{~min}$ in a Beckman Optima TLX ultracentrifuge with a TLA100.3 rotor with the brake turned off, or in a Beckman Optima LE- 80 ultracentrifuge with Ti 50.4 rotor at $172,000 \times g$ for $40 \mathrm{~min}$ with the brake off and the deceleration set to zero. Centrifugation at this step was important for the success of the procedure, taking care that the MTs were not pelleted too hard or subjected to hard braking. The pellet, containing the MT fraction, was gently resuspended in $80 \mathrm{~mm}$ PIPES, pH 6.8, $1 \mathrm{~mm} \mathrm{MgCl}_{2}$ at room temperature, then frozen in liquid nitrogen.

When MT-containing isolates were thawed, $2.2 \mathrm{~mm}$ dithiothreitol, 1.1 mM GTP, and phosphatase and kinase inhibitors were added. Tauassociated MTs were isolated by immunoprecipitating with Tau-5 (EMD Chemicals)-conjugated beads, followed by isolation of the MAP-2 associated MTs with AB5392 (Abcam)-conjugated beads. Beads were washed with $80 \mathrm{~mm}$ PIPES, pH 6.8, plus $1 \mathrm{~mm} \mathrm{MgCl}_{2}$, followed by elution with 80 mм PIPES, pH 6.8, 1 mм $\mathrm{MgCl}_{2}$, and $1 \mathrm{M}$ sodium chloride. The remaining unbound MTs have previously been shown to be largely cold stable MTs (Fanara et al., 2010).

Tubulin was purified from these immunoprecipitation extracts using size exclusion chromatography (Biosep SEC-S2000 column, Phenomenex). Tubulin samples were hydrolyzed with $6 \mathrm{~N}$ hydrogen chloride at $110^{\circ} \mathrm{C}$ for $16 \mathrm{~h}$. Amino acids were derivatized to form pentafluorobenzyl derivatives for gas chromatography/mass spectrometry analysis and ${ }^{2} \mathrm{H}-$ incorporation into alanine released from total tubulin protein was measured. ${ }^{2} \mathrm{H}$-enrichment was calculated as the percentage increase, over natural abundance, in the percentage of alanine derivative present as the $(\mathrm{M}+1)$ mass isotopomer (EM1). The ${ }^{2} \mathrm{H}_{2} \mathrm{O}$ body water enrichment for each animal was determined from plasma to allow the final calculation of the fraction of ${ }^{2} \mathrm{H}$-labeled tubulin in the MT preps. Measurement of ${ }^{2} \mathrm{H}_{2} \mathrm{O}$ enrichment in body water was measured using a modification of previously described procedures (Lis et al., 2008). Briefly, plasma samples were centrifuged at $500 \times g$ for $10 \mathrm{~min}$ at $4^{\circ} \mathrm{C}$ to eliminate cells and other insoluble material. Aliquots of $1 \mathrm{ml}$ were collected in cryogenic polypropylene tubes, fast frozen in liquid nitrogen, and stored at $-80^{\circ} \mathrm{C}$. Aliquots of plasma were freshly thawed on ice, diluted 1:100, and placed into the caps of inverted sealed screw-capped vials for overnight distillation at $80^{\circ} \mathrm{C}$. Protons from plasma water were transferred to acetylene by reaction with calcium carbide. Acetylene samples were then analyzed using a Series 3000 cycloidal mass spectrometer (Monitor Instruments), which was modified to record ions at mass/charge ratios of 26 and 27 (M0 and $\mathrm{M} 1$ ) and calibrated against a standard curve prepared by mixing 99.9\% ${ }^{2} \mathrm{H}_{2} \mathrm{O}$ with unlabeled water.

We observed that the tau-associated MT fraction from $\mathrm{rTg} 4510$ mice was not as stable as that from other mice or as the MAP-2-associated MT fractions. Studies in which the average coefficient of variability within groups was $>30 \%$ were considered failed. In those failed studies, the differences between rTg4510 and tTA Alone control samples were diminished or absent. In 16 studies where tau-associated MTs were measured from $\mathrm{rTg} 4510$ mice, 6 failed (38\%). MAP-2-associated MTs were measured from 11 studies, and only 1 (9\%) failed. In three studies where tau-associated MT variability was high, two had acceptable MAP-2associated MT variability. Control experiments were performed with MTs from tau knock-out mice, and no detectable signal was observed in the MT fraction isolated with the Tau-5 immunoprecipitation. We suspect this variability in tau MTs, which has not been observed in other transgenic mouse lines, was due to lower stability of those MTs during the isolation procedure from the very high expression of tau in the rTg4510 mice (Fig. 1).

Western blot analyses. Western blot analyses were performed as described previously (Fanara et al., 2007, 2010) using Tau5 to detect all forms of tau, ab5392 to detect MAP-2, and DM1A to detect $\alpha$-tubulin (Sigma-Aldrich). Equal amounts of protein (BCA Protein Assay, Thermo Scientific) were loaded in each lane, and quantitation of $\alpha$-tubulin was done using densitometry of film in the linear range. The amount of tubulin in each sample was normalized to a standard curve comprised of serial dilutions of purified tubulin (catalog \#TLA238, Cytoskeleton) and a negative control (no tubulin), represented as $0 \%$.

Morris water maze. Mice were trained in the Morris water maze (MWM) for $6 \mathrm{~d}$ at 2.5 months of age, before any treatment. The mice 

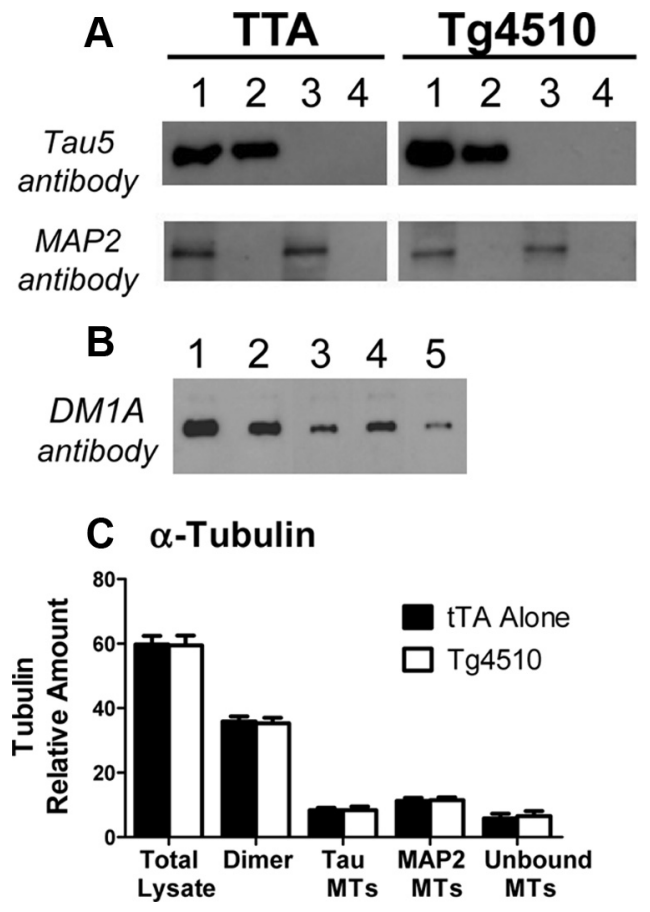

Figure 1. hTau-P301L overexpression in Tg4510 mice does not alter MT isolation or tubulin distribution in brain tissue. $A, M T$ isolation procedure by sequential immunoprecipitation. Lane 1, total lysate; Lane 2, isolated tau MTs; Lane 3, isolated MAP-2 MTs; Lane 4, unbound (coldstable) MTs. $\boldsymbol{B}$, Western blot of $\alpha$-tubulin from MT isolation: Lane 1, total lysate; Lane 2, dimer fraction; Lane 3, isolated tau MTs; Lane 4, isolated MAP-2 MTs; Lane 5, unbound MTs. C, Quantitation of $\alpha$-tubulin Western blots, with an $n=4$ for unbound MTs and $n=8$ for all other groups.

were distributed into treatment groups based on the results of the behavioral analysis using the rank scores for the probe trial annulus crossing memory index to make sure there were no performance issues or significant memory bias between the experimental and control groups before dosing. Dosing with BMS-241027 began $4 \mathrm{~d}$ after the completion of the behavioral analysis. Mice were tested again in the MWM after the eighth dose, at 4.5 months of age. The second round of water maze testing was performed in another testing room to avoid carrying-over or savings effects. Mice were acclimated to the experimental room for 2-3 d before testing. The mice were placed in a water maze of $1.5 \mathrm{~m}$ diameter, with a 16 -cm-diameter platform placed $0.5 \mathrm{~cm}$ under the surface of the water. The water was made opaque with nontoxic white tempera paint, and the water temperature was kept at $25^{\circ} \mathrm{C}$. There were four training trials per day of up to $90 \mathrm{~s}$ each, with a $10 \mathrm{~s}$ post-trial period on the platform after each. Mice were placed under a heat lamp to dry after each trial. The interval between trials ranged from 25 to $35 \mathrm{~min}$. The search path of mice was tracked using HVS Image Advanced Tracker HVA2020 software. Acquisition training was performed for 5 consecutive days, with a $1 \mathrm{~min}$ probe trial $18 \mathrm{~h}$ after the last training trial. During probe trials, the platform was removed from the pool. Swim speed and float time (swim speeds of $<5 \mathrm{~cm} / \mathrm{s}$ ) was recorded for all animals and did not change with drug treatment (data not shown).

Immunohistochemistry and Gallyas silver staining. Immunohistochemistry on paraffin-embedded sections was performed as described previously (Barten et al., 2011). AT8 and AT180 were obtained from Thermo Scientific, anti-pTau181 antibody Anaspec, MC-1 from Peter Davies (Albert Einstein College of Medicine, Bronx, NY), anti-GFAP polyclonal antibody from Dako, and anti-Ibal polyclonal antibody from Wako. A subjective scoring system was developed for each tau antibody based on the distribution in hippocampus and cortex, and the extent of staining in neuronal soma and dendrites. The pattern differed for each antibody. Four sections were scored for each animal with blinding to treatment. Silver staining was performed using the Gallyas method. A semiquantitative subjective scoring system was developed based on relative numbers of silver-positive cells per section, analyzing four sections per animal. Silver stain scoring was based on the numbers of positive neurons, not intensity, which can vary between sections. After deparaffinization, $5 \mu \mathrm{m}$ sections were incubated in $5 \%$ periodic acid for $5 \mathrm{~min}$, washed in water, and then placed in alkaline silver iodide solution (containing 1\% silver nitrate) for $1 \mathrm{~min}$. The sections were then washed in $0.5 \%$ acetic acid for $10 \mathrm{~min}$, placed in developer solution for $15 \mathrm{~min}$, before washing with $0.5 \%$ acetic acid, then water. The sections were then treated with $0.1 \%$ gold chloride for 5 min before washing in water, and incubation in 1\% sodium thiosulphate (hypo) for $5 \mathrm{~min}$, before a final wash and counterstaining with $0.1 \%$ nuclear fast red.

Hippocampal cell quantitation. Brains were cut sagittally between the midline and $\sim 0.75 \mathrm{~mm}$ laterally at $5 \mu \mathrm{m}$. Nissl-stained slides were scanned and digitized using the Aperio ScanScope. Images of the entire brain section were captured at high resolution and were stored as files within Spectrum (Aperio Technologies). To process images, a region of $4000 \times 4000$ pixels, including the entire hippocampus, was captured using the extract tool and saved as a JPEG file for importing into MetaMorph (Molecular Devices) for quantification of cell loss within the CA1 and $\mathrm{CA} 3$ regions of the hippocampus. Cells within a randomly placed set of boxes over the hippocampus were counted. The same regions were used for every image, and five sections were counted per animal, five slides apart.

Statistical analyses. Statistical analyses were performed with ANOVA followed by Dunnett's multiple-comparison test using Graphpad Prism 5 , unless indicated otherwise. For analysis of semiquantitative histopathological scores, the Kruskal-Wallis test was used. Statistical analyses of data with two or fewer samples per group were performed using ANOVA with a Bonferroni's correction and pooled estimate of error using SAS, version 9.1. MWM was analyzed by two-way ANOVA followed by Tukey's post hoc tests using Sigmaplot version 11 .

\section{Results}

\section{Altered MT dynamics in tau transgenic mice}

MT dynamics can be measured in vivo using a novel deuterium labeling procedure (Fanara et al., 2004, 2007, 2010). Following labeling of body fluids with ${ }^{2} \mathrm{H}_{2} \mathrm{O}$, deuterium becomes incorporated into nonessential amino acids and then into newly synthesized proteins. The kinetics and extent of ${ }^{2} \mathrm{H}$-alanine incorporation into soluble tubulin dimers and exchangeable tubulin polymers will be equal in most cells due to the very rapid equilibrium between these tubulin pools (Fanara et al., 2004). However, in neurons, the dynamic exchange of newly synthesized ${ }^{2} \mathrm{H}$-tubulin dimers into MTs is reduced due to higher MT stability (Fanara et al., 2004, 2010). Using this method, MT dynamics were measured in the cortex of $\mathrm{rTg} 4510$ mice. In these studies, MTs were purified to three fractions: the tau-associated MTs (normally axonal), MAP2-associated MTs (normally dendritic), and the unbound fraction, which mostly represents cold stable MTs (normally in the axon hilus) (Fanara et al., 2010). Even though there is a 13-fold overexpression of tau in rTg4510 mice (Santacruz et al., 2005), the MT isolation procedure showed no cross-contamination of tau or MAP-2 between fractions (Fig. $1 A)$. Interestingly, there were no differences in the amount of $\alpha$-tubulin measured in the dimer or MT fractions between tTA Alone and rTg4510 mice (Fig. $1 B, C$ ). Even so, there was an agedependent increase in the dynamic exchange of ${ }^{2} \mathrm{H}$-tubulin dimers into MTs in cortex of $\mathrm{rTg} 4510$ mice in all MT fractions measured (Fig. $2 A-C$ ). At 5 months of age, only the rTg4510 mice showed increased exchange of ${ }^{2} \mathrm{H}$-tubulin dimers into MTs when compared with mice containing only the transactivator gene (tTA Alone), the tau gene (Tau Alone), or neither (Dble Neg) (Fig. 2D). Tau-P301L transgene expression can be reduced $75 \%$ in rTg4510 mice with doxycycline treatment (Santacruz et al., 2005; Barten et al., 2011), which was also observed in this study by 
immunohistochemical staining of tau (Fig. 3A). Doxycycline treatment for 3 months in rTg4510 mice reduced MT hyperdynamicity to the level of control mice (Fig. 3B).

Because $\mathrm{rTg} 4510$ is a very aggressive tauopathy model, we also examined $3 \times \mathrm{Tg}$ mice, which have a much slower and milder phenotype. Measurements were made from the hippocampus, where the phospho-tau ( $\mathrm{p}$-tau) pathology is concentrated. 3xTg MTs were hyperdynamic in the tau-associated fraction only at 20 months of age (Fig. 2E), and were not significantly altered at any age in the MAP-2-associated fraction (data not shown). The unbound fraction was only measured at 20 months of age $(n=2)$, where it was significantly increased from an average of 4.3 to $8.9 \%$ newly synthesized tubulin for $\mathrm{C} 57 \mathrm{BL} / 6$ and $3 \mathrm{xTg}$ mice, respectively. Together, these studies suggest an association between tau-P301L transgene expression and pathology with altered MT turnover.

\section{BMS-241027 reduced MT \\ hyperdynamicity in rTg4510 \\ mice to baseline levels}

BMS-241027, a brain-penetrant MT stabilizer with a long half-life in brain and a short half-life in blood (Andrieux et al., 2006; Brunden et al., 2010), was administered to rTg4510 mice. BMS-241027 has previously been used to reduce tumor formation in nude mice with $15-35 \mathrm{mg} / \mathrm{kg}$, i.p., treatment every other day for five cycles for a cumulative dose of 75-175 mg/kg over $10 \mathrm{~d}$ (Kolman, 2004). Because the compound has a brain half-life of $\sim 48 \mathrm{~h}$ in mice (unpublished observations), it would accumulate significantly in brain with every-other-day dosing. We chose cumulative doses that were $\sim 10$ - and 100-fold lower than oncology doses by administering the compound once weekly at 1 and $10 \mathrm{mg} / \mathrm{kg}$, i.p. rTg4510 mice were dosed for 3 months to determine whether MT hyperdynamicity could be mitigated. In the cortex, where there is high tau-P301L transgene expression and pathology, hyperdynamic tau-associated, MAP-2-associated and unbound MTs were reduced in a dose-dependent manner to the level observed in tTA Alone animals (Fig. $4 A-C$ ). While there was a $350 \%$ elevation of MT dynamics in cortex of $\mathrm{rTg} 4510$ mice, there was only a $30 \%$ increase in cerebellum, consistent with very low levels of transgene expression there. BMS-241027 treatment in this study reduced MT dynamicity to baseline levels, and no lower, in both brain regions. This suggests that BMS-241027 had a preferential beneficial effect on hyperdynamic MTs (Fig. 4D). rTg4510 mice were treated with BMS-241027 for shorter time periods to determine when reductions in MT hyperdynamicity in cortex would be observed. Surprisingly, we did not observe reductions in hyperdynamic MTs after 2 or 8 weeks of treatment, even though we repeatedly observed changes following 11 weeks of treatment (Fig. 5). These studies demonstrated that BMS-241027 only altered exchange of ${ }^{2} \mathrm{H}$-tubulin dimers into MTs following an extended treatment period.

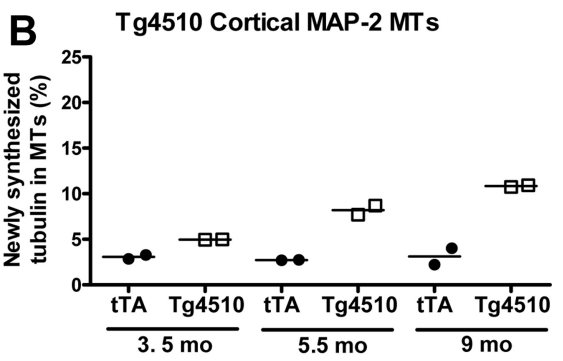

C Tg4510 Cortical Unbound (CS) MTs

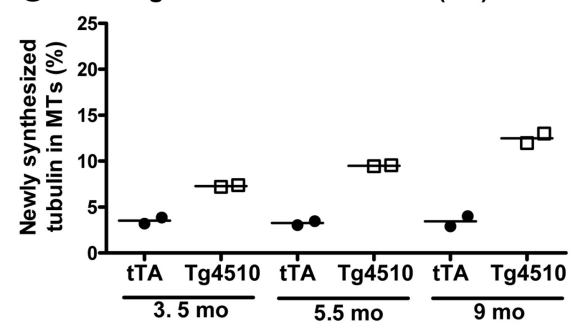

E $3 \times 19$ Hippocampal Tau MTs

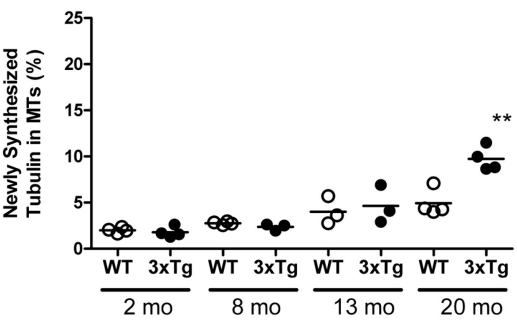

Figure 2. MT hyperdynamicity in rTg4510 and 3xTg tauopathy models. $r T g 4510$ mice were of mixed sex, and 3xTg mice were females compared with age-matched $(57 \mathrm{BL} / 6 \mathrm{~J}$ mice. Cortical MTs from Tg4510 mice were examined in $\boldsymbol{A}-\boldsymbol{D}$, and hippocampal MTs from 3xTg mice in $E .{ }^{* *} p<0.01,{ }^{* *} p<0.001$.

BMS-241027 treatment prevented cognitive deficits and pathology in rTg4510 mice

rTg4510 mice are known to develop cognitive deficits between the ages of 2.5 and 4.5 months of age (Santacruz et al., 2005). Because the onset of these deficits can vary, mice were tested in the MWM at 2.5 months of age to screen for group bias before dosing (Fig. 6A). The mice were tested again after 2 months of treatment with BMS-241027 to analyze changes in MWM performance. Vehicle-treated animals no longer learned the position of the hidden platform, while $\mathrm{rTg} 4510$ mice treated with $1 \mathrm{mg} / \mathrm{kg}$ BMS-241027 were able to learn the location of the hidden platform (Fig. $6 \mathrm{~B}$ ). In the probe trial, mice treated with both 1 and 10 $\mathrm{mg} / \mathrm{kg}$ BMS-241027 showed significantly more time in the target quadrant than the vehicle-treated mice, indicating that they remembered the location of the platform (Fig. $6 C$ ). The vehicletreated animals did not show any preference for the target quadrant, spending equal amounts of time in all quadrants.

Following behavioral testing, the mice were treated until they were 5.5 months of age, when severe neurodegeneration is observed in the hippocampus. An age-dependent increase of intraneuronal p-tau accumulation, tangle formation, and neurodegeneration has been well described in rTg4510 mice (Ramsden et al., 2005; Santacruz et al., 2005; Spires et al., 2006). The $1 \mathrm{mg} / \mathrm{kg}$ BMS-241027 treatment group showed a significant reduction in tau pathology, as measured by immunohistochemical staining for AT8 (p-tau $202+205)$, AT180 (pTau 231), and p-tau 181, with a trend for the conformation-specific antibody MC1 (Figs. 7, 8). Tangle formation, as measured by Gallyas silver stain, was also reduced in the $1 \mathrm{mg} / \mathrm{kg}$ treatment group in multi- 


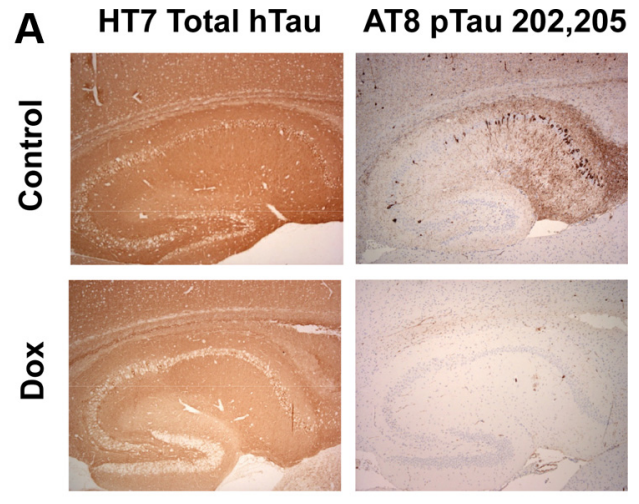

B Tau MTs

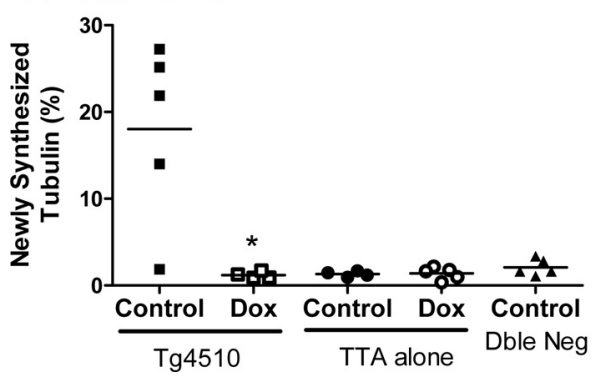

Figure 3. $\quad \boldsymbol{A}, \boldsymbol{B}$, Tau transgene reduction reduced tau protein expression $(\boldsymbol{A})$ and reduced or prevented MT hyperdyamicity $(\boldsymbol{B})$. Male mice were treated with doxycycline (Dox) in their chow from 2.5 to 5.5 months of age. Note: The rTg4510 mouse with low MT dynamicity in the control chow case was genotype confirmed. It may represent variability in the phenotype, as we have occasionally seen rTg4510 mice with little tau pathology, or it may represent variability in the assay. This is the only study included where the average coefficient of variation was $>30 \%$. ${ }^{*} p=0.02$ using a Student's $t$ test. ple brain regions, with little or no benefit in the $10 \mathrm{mg} / \mathrm{kg}$ group (Figs. 7, 8). In addition, there were significantly more CA1 and CA3 neurons in the $1 \mathrm{mg} / \mathrm{kg}$ treatment group, similar to the numbers measured in nontransgenic control mice (Fig. $8 C$ ).

In these pathological measures, a greater treatment effect was observed in the $1 \mathrm{mg} / \mathrm{kg}$ group than the $10 \mathrm{mg} / \mathrm{kg}$ group. This suggests that there may be a U-shaped dose-response curve for some of the endpoints measured in this study. No toxicity was observed (weight loss, or behavioral or histopathological toxicity) in either 1 or $10 \mathrm{mg} / \mathrm{kg}$ groups in 2 and 3 month dosing studies in these mice, suggesting that overt toxicity was not responsible for an apparent U-shaped dose-response curve.

\section{Discussion}

It is difficult to measure MT dynamics in the brain, where MTs are more abundant and more stable than in any other tissue. We could not measure reproducible changes of MT mass or total or acetylated tubulin in brain tissue to indicate alterations from tauP301L transgene expression or BMS-241027 treatment (Fig. 1; unpublished observations). This is consistent with data from others showing the lack of measureable effect on MT mass following tau hyperphosphorylation in vivo (Planel et al., 2008) and maximal tubulin acetylation in brain tissue (Brunden et al., 2011). Interestingly, Brunden et al. (2011) were able to measure increases in tubulin acetylation in the optic nerves of mice following acute BMS-241027 treatment. Although small decreases in the number of MTs as measured by electron microscopy and inhibition of transport function in nerves have been described in tauopathy models (Zhang et al., 2005, 2012), alterations in MT turnover due to tau abnormalities have not been previously described in vivo. Using a stable isotope-labeling technique, we have shown that MTs isolated from both rTg4510 and 3xTg tauopathy models dynamically exchanged more soluble tubulin dimers with MTs in vivo than control mice. Thus, tau pathology is associated with a larger pool of dynamic MTs in brain. This result would not have necessarily been predicted. Although tau-P301L is less efficient at binding to MTs than endogenous tau, it does have MT-stabilizing capability in neuronal cultures (Dayanandan et al., 1999). Therefore, although not observed, it is conceivable that high tau levels would have been able to compensate for reduced activity.

Abnormal MT turnover in the two transgenic lines was generally consistent with the level of tau pathology not just total tau protein levels. 3xTg mice have approximately twofold overexpression of the tau-P301L transgene and much less pathology than the 13-fold overexpressing rTg4510 line (Santacruz et al., 2005; Barten et al., 2011). Even so, Tau Alone transgenic mice have leaky transgene expression from the TRE promoter that is close to the amount observed in $3 \times \mathrm{Tg}$ mice. Tau Alone mice do not have either abnormal tau accumulation (Barten et al., 2011) or hyperdynamic MTs (Fig. 2D). Furthermore, doxycycline treatment of rTg4510 mice completely reversed MT hyperdynamicity (Fig. 3) and greatly re-
Figure 4. BMS-241027 reduced MT hyperdynamicity in rTg4510 mice following 3 months of treatment. $\boldsymbol{A}$, Tau-associated MT results are representative of two studies, with a third study failing to meet quality control criteria as described in Materials and Methods. $\boldsymbol{B}$, MAP-2-associated MT results are representative of all three studies. $\boldsymbol{C}, \boldsymbol{D}$, Cold stable MTs from cortex and tauassociated MTs from cerebellum were only measured from one small study. $\boldsymbol{A}$ and $\boldsymbol{B}$ used female mice, and $\boldsymbol{C}$ and $\boldsymbol{D}$ used mixed sexes. mpk $=\mathrm{mg} / \mathrm{kg} ;{ }^{*} p<0.05,{ }^{* *} p<0.01,{ }^{* * *} p<0.001$.
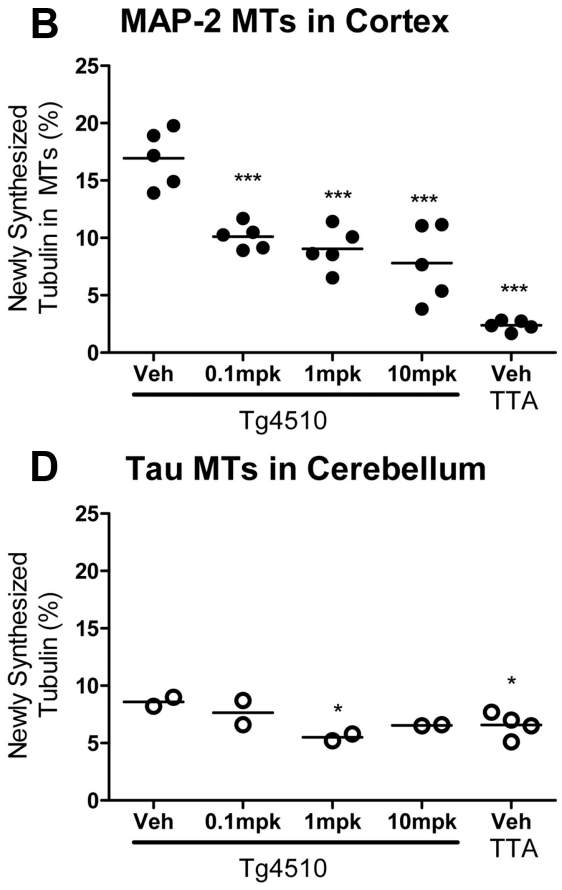


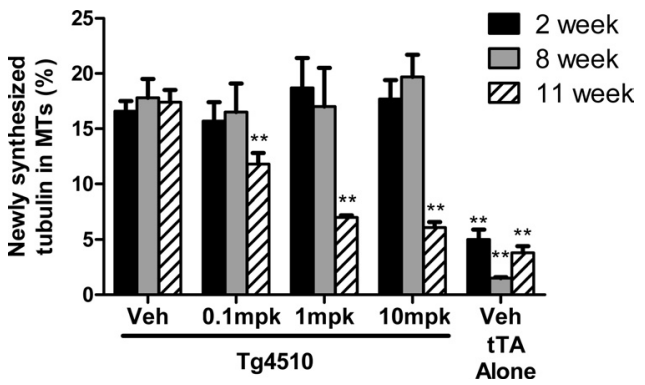

Figure 5. BMS-241027 had no effect on Tau-associated MT hyperdynamicity following 2 or 8 weeks of treatment. $n=5-9$ per group, and sexes are mixed. Data from 2 and 11 week treatments were combined from two studies. ${ }^{* *} p<0.01$. Veh, Vehicle.

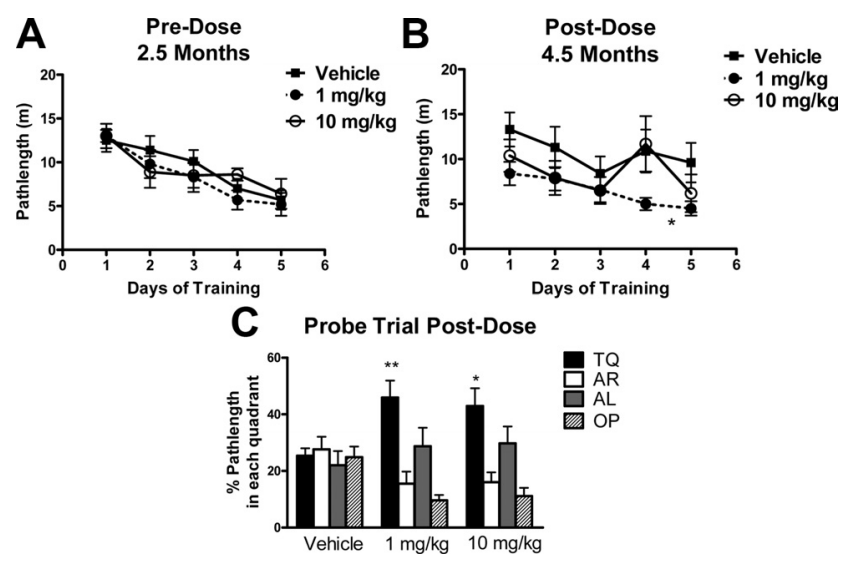

Figure 6. BMS-241027 prevented cognitive deficits in male rTg4510 mice. A, Before dosing at 2.5 months of age, there were no differences between groups of rTg 4510 mice in their ability to learn the location of a hidden platform in the MWM. $\boldsymbol{B}$, After 2 months of epothilone treatment, the $1 \mathrm{mg} / \mathrm{kg}$ treatment group was significantly different from vehicle control in the acquisition phase. C, Both 1 and $10 \mathrm{mg} / \mathrm{kg}$ treatment groups showed significant differences from vehicle in the probe trial. TQ, Target; AR, adjacent right; AL, adjacent left; OP, opposite quadrant. The $n$ per group was 7,9 , and 6 for vehicle, $1 \mathrm{mg} / \mathrm{kg}$ and $10 \mathrm{mg} / \mathrm{kg}$ treatment groups, respectively. Larger groups were initially used, but only data from males are shown to reduce any sex differences in the data. Data were analyzed by two-way ANOVA with significant differences in treatment and day for acquisition data and significant differences in quadrants and treatment for probe data. Treatment differences are indicated on the plots. ${ }^{*} p<0.05,{ }^{* *} p<0.01$.

duced the accumulation of AT8+ neurons (Fig. 2A), even though soluble tau protein remains higher than that of very old 3xTg mice (Santacruz et al., 2005; Barten et al., 2011). Although the $3 \times \mathrm{Tg}$ colony at BMS has very little plaque load, the addition of amyloid precursor protein and presenilin-1 mutant protein expression to the tau-P301L expression may also have contributed to the altered MT hyperdynamicity observed in this line. Even so, we also observe an exponential increase in Sarkosyl-insoluble tau accumulation and AT8+ neurons between 17 and 23 months in 3xTg mice (Barten et al., 2011), which may contribute to the significant increase in MT dynamics observed here at 20 months (Fig. 2E). Together, these results suggest that pathological forms of tau are associated with changes in MT turnover in vivo.

There are multiple possible mechanisms whereby pathological forms of tau could lead to increased MT dynamicity in vivo. It is noteworthy that in addition to tau MTs, MAP-2 and cold stable MTs were also affected by the accumulation of pathological tau. It is possible that high concentrations of human tau-P301L interfere with the ability of endogenous tau, and possibly even MAP-2 and STOP (stable tubule only peptide), to stabilize MTs as has been described in vitro (Alonso et al., 1996; Mocanu et al., 2008).
There may be downstream effects of tau that could influence MT stability, such as alterations in stathmin phosphorylation (Manna et al., 2009), ATP and GTP levels, or the activity of the wide array of proteins now known to regulate MTs at their tip ends (Jiang and Akhmanova, 2011). Tau phosphorylation helps determine the spacing between microtubules, and so alterations could influence the transport of larger cargo, such as mitochondria (Shahpasand et al., 2012), significantly impacting neuronal function through changes in ATP availability. Tau mislocalization has been associated with disruption of neurofilament structure, loss of dendritic spines (Zempel and Mandelkow, 2011), and alterations in AMPA receptor trafficking (Hoover et al., 2010). Tau is known to be involved in the transport of Fyn to the dendrites where it plays a role in NMDA receptor cycling (Ittner and Gotz, 2011) and in BDNF signaling (Chen et al., 2012). This and other roles of tau in cell signaling (Morris et al., 2011) could indirectly lead to changes in MT dynamics, including for MAP-2 and cold stable MTs (Job et al., 1983; Montoro et al., 1993; Fanara et al., 2010). It is also possible that pathological forms of tau lead to a reduction in the average length of MTs, which would then result in more free MT ends, and more opportunities to exchange with the labeled tubulin dimers. This could occur if MT severing enzymes such as katanin or spastin were more active in the presence of abnormal tau. In fact, normal tau has been shown to protect MTs against katanin cleavage (Qiang et al., 2006). It is likely that the accumulation of abnormal forms of tau will have pleiotropic effects in neurons.

In rTg4510 mice, low doses of BMS-241027 restored MT turnover to basal levels as measured with this technique. This reduced dynamicity was observed in both the cortex, where transgene expression and hyperdynamicity were high, and the cerebellum, where transgene expression and hyperdynamicity were low. These data suggest that hyperdynamic MTs are preferentially affected by BMS-241027 at low doses. In contrast, oncologic doses of paclitaxel are able to reduce ${ }^{2} \mathrm{H}$-label incorporation below basal levels into tumor cell MTs, which are very dynamic (Fanara et al., 2004). BMS-241027 reduced MT hyperdynamicity in rTg4510 mice with a typical dose-response curve and a maximal effect near the $10 \mathrm{mg} / \mathrm{kg}$ dose.

On the other hand, the optimal BMS-241027 dose to reduce pathological deficits in $\mathrm{rTg} 4510$ mice was $1 \mathrm{mg} / \mathrm{kg}$ with no significant benefit at $10 \mathrm{mg} / \mathrm{kg}$. Only partial reduction of hyperdynamic MTs was observed in rTg4510 mice at the $1 \mathrm{mg} / \mathrm{kg}$ dose. MT-stabilizing agents are known to influence MT dynamics at molar ratios as low as 1:1000 through allosteric effects, so it is reasonable to expect pharmacological activity at much lower doses than that required for inhibition of mitosis (Derry et al., 1995). It is not surprising that an apparent U-shaped dose response is observed with BMS-241027, as there is likely an optimal range of MT stabilization for maximal neuronal function. These data were consistent with the "dosage effect" model previously described for MT support of long-term viability (Feinstein and Wilson, 2005). In addition, U-shaped dose-response curves have been described for paclitaxel in the stimulation of nuclear transport (Giannakakou et al., 2002), the enhancement of neurite outgrowth (Sengottuvel et al., 2011), and the reduction of pathology and transport deficits induced by tau-P301L transfection (Shemesh and Spira, 2011). Although it may be expected that optimal neuronal function would be achieved when MT dynamics in rTg4510 neurons were reduced to the same level as control mice (as at $10 \mathrm{mg} / \mathrm{kg}$ ), those neurons may differ in their optimal set point due to the transgene expression. There was no evidence that loss of activity with the $10 \mathrm{mg} / \mathrm{kg}$ dose was due to toxicity. 

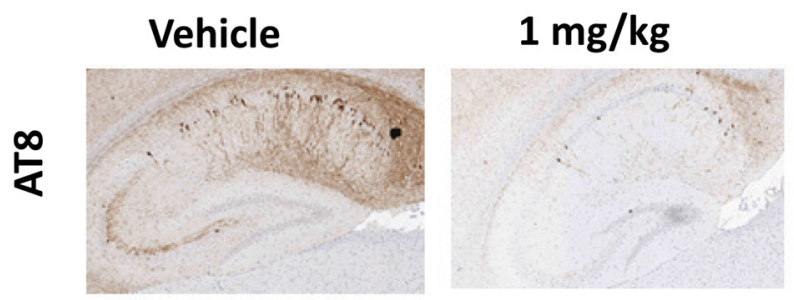

$10 \mathrm{mg} / \mathrm{kg}$
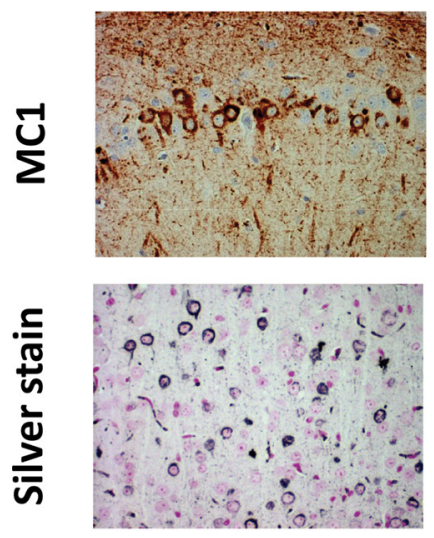
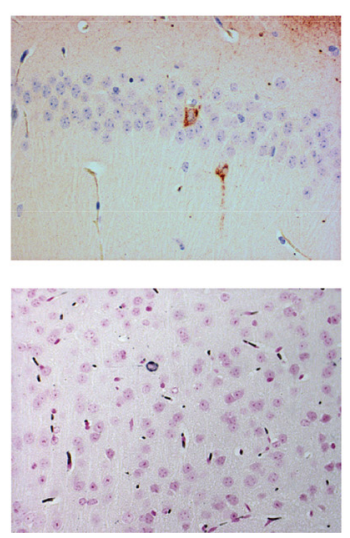
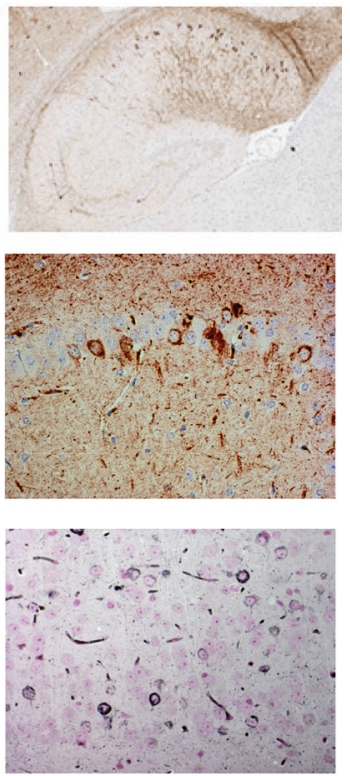

Figure 7. BMS-241027 treatment for 3 months reduced tau pathology in male $\mathrm{rTg} 4510$ mice.

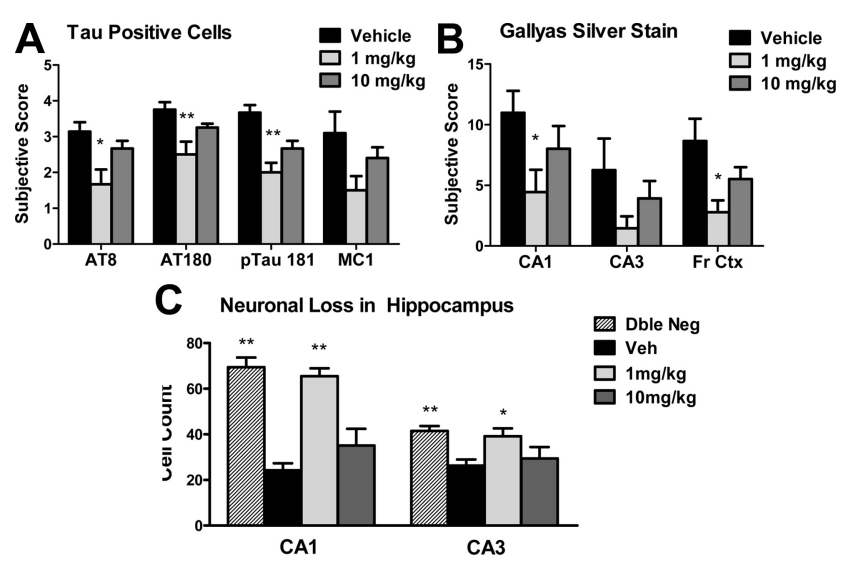

Figure 8. BMS-241027 treatment for 3 months reduced tau pathology and neuron loss in male rTg4510 mice. A, Quantitation of Tau immunohistochemical staining. B, Quantitation of Gallyas silver stain. C, Quantitation of neuronal number in hippocampus. ${ }^{*} p<0.05,{ }^{* *} p<$ 0.01. Dble, Double; Veh, vehicle.

The pathological and functional benefits observed with 1 $\mathrm{mg} / \mathrm{kg}$ BMS-241027 in rTg4510 mice are consistent with results obtained in the PS19 tauopathy model. Epothilone D (BMS241027) at $1-3 \mathrm{mg} / \mathrm{kg}$ reduces dystrophic neurites, restores MT numbers, and improves performance in the Barnes maze in a 3 month prevention trial (Brunden et al., 2010). Subsequent studies also show beneficial effects of Epothilone D at 0.3 and $1 \mathrm{mg} / \mathrm{kg}$ in a 3 month interventional trial (Zhang et al., 2012). Epothilone D-treated mice had improvements in fast axonal transport in optic nerve, Barnes maze and Y maze deficits, tau pathology, and neurodegeneration. We performed a study in a third tauopathy model by treating $3 \times \mathrm{Tg}$ mice with $0.1,1$, and $10 \mathrm{mg} / \mathrm{kg}$ BMS241027 from 6 to 12 months of age. Although previous studies with the BMS colony of 3xTg mice had demonstrated deficits in MWM at 12 months, in this study the 3xTg mice performed significantly better than vehicle-treated wild-type control mice. In addition, there was no difference in performance between vehicle and BMS-241027 treated 3xTg mice (data not shown).
These data suggest that low doses of BMS241027 do not have effects on cognition in the absence of a disease state.

An unexpected finding was that 3 months of BMS-241027 treatment were required to reduce the elevation in MT turnover in rTg4510 mice. Two months of treatment was without effect on MT dynamics as measured using this assay, even though cognitive benefits were observed after 2 months and at the same age. This finding was even more surprising because changes in MT turnover can be measured in response to a fear-conditioning protocol within $24 \mathrm{~h}$ with this method (Fanara et al., 2010). In addition, increases in acetylated tubulin can be observed following a single dose of BMS-241027 (Brunden et al., 2011). A likely explanation is that low doses of BMS-241027 cause small changes in MT stability that do not translate to measurable changes in the pool of dynamic MTs measured with this assay. The changes eventually observed following 3 months of dosing are likely due to compensatory changes in MTs that can then be measured with the stable isotope-labeling technique. There are several scenarios consistent with this idea. In one hypothesis, tau dysfunction leads to an accumulation of shorter MTs, and these smaller MTs exist in nonparallel orientations that hinder transport, as has been observed in Aplysia neurons transfected with tau-P301L (Shemesh and Spira, 2010). With time, these shorter MTs would eventually be replaced by longer and properly oriented MTs. A second scenario could involve an direct antiinflammatory effect in glia, which would then help neurons to cope better with the stress of overexpressing high levels of tau$\mathrm{P} 301 \mathrm{~L}$. The MTs measured in this assay are predominantly neuronal due to the specific isolation of MTs with tau and MAP-2 bound to them and the much greater enrichment of MTs in neurons compared with other cell types (Dehmelt and Halpain, 2005; Ittner et al., 2011). A direct effect of BMS-241027 on glia would be consistent with the anti-inflammatory response reported in two axonal injury models following paclitaxel treatment (Hellal et al., 2011; Sengottuvel et al., 2011). A third scenario could involve stabilization of a small population of MTs that turn over so rapidly that they cannot be isolated or measured within the larger pool of MTs measured with this assay. MTs have been imaged moving in and out of dendritic spines in the seconds to minutes timeframe, and are hypothesized to play a role in spine alterations in response to activity (Hoogenraad and Akhmanova, 2010). Finally, BMS-241027 may be improving neuronal function through an influence on signaling pathways, protein clearance, or mitochondrial function, which would then lead to later improvements in MT dynamics (Michaelis et al., 2005; Shemesh et al., 2008; Vossel et al., 2010; Gardiner et al., 2011; Lee et al., 2011; Silva et al., 2011).

MT hyperdynamicity, along with deficits in slow axonal transport, has also been measured in a model of amyotrophic lateral sclerosis with this stable isotope-labeling technique (Fanara et al., 2007). No changes in total tubulin or MTs are observed, demonstrating another neurodegeneration model where in vivo changes in MT turnover would not have been detected without stable isotope labeling. Treatment of these mice for 3 months with 
noscapine, a microtubule modulator, is associated with improved motor performance, increased lifespan, and reduced neurodegeneration. These data suggest that tau alterations are not the only mechanism that can induce an increase in MT turnover in vivo and that MT stabilization may have broader utility in neurodegenerative disease.

In summary, we have demonstrated that MT hyperdynamicity is associated with the accumulation of pathological forms of tau in transgenic mice. Treatments that reduced tau-P301L transgene expression or stabilized MTs were able to restore normal MT dynamics. When MTs were stabilized at very low doses, reductions in tau pathology, neuronal loss, and cognitive deficits were observed. These data suggest that MT stabilization could be useful as a therapeutic approach for the treatment of $\mathrm{AD}$ and other tauopathies.

\section{References}

Alonso AC, Grundke-Iqbal I, Iqbal K (1996) Alzheimer's disease hyperphosphorylated tau sequesters normal tau into tangles of filaments and disassembles microtubules. Nat Med 2:783-787.

Andrieux A, Salin P, Schweitzer A, Bégou M, Pachoud B, Brun P, Gory-Fauré S, Kujala P, Suaud-Chagny MF, Höfle G, Job D (2006) Microtubule stabilizer ameliorates synaptic function and behavior in a mouse model for schizophrenia. Biol Psychiatry 60:1224-1230.

Barten DM, Cadelina GW, Hoque N, DeCarr LB, Guss VL, Yang L, Sankaranarayanan S, Wes PD, Flynn ME, Meredith JE, Ahlijanian MK, Albright CF (2011) Tau transgenic mice as models for cerebrospinal fluid tau biomarkers. J Alzheimers Dis 24 [Suppl 2]:127-141.

Beer TM, Higano CS, Saleh M, Dreicer R, Hudes G, Picus J, Rarick M, Fehrenbacher L, Hannah AL (2007) Phase II study of KOS-862 in patients with metastatic androgen independent prostate cancer previously treated with docetaxel. Invest New Drugs 25:565-570.

Brunden KR, Trojanowski JQ, Lee VM (2009) Advances in tau-focused drug discovery for Alzheimer's disease and related tauopathies. Nat Rev Drug Discov 8:783-793.

Brunden KR, Zhang B, Carroll J, Yao Y, Potuzak JS, Hogan AM, Iba M, James MJ, Xie SX, Ballatore C, Smith AB 3rd, Lee VM, Trojanowski JQ (2010) Epothilone D improves microtubule density, axonal integrity, and cognition in a transgenic mouse model of tauopathy. J Neurosci 30:13861-13866.

Brunden KR, Yao Y, Potuzak JS, Ferrer NI, Ballatore C, James MJ, Hogan AM, Trojanowski JQ, Smith AB 3rd, Lee VM (2011) The characterization of microtubule-stabilizing drugs as possible therapeutic agents for Alzheimer's disease and related tauopathies. Pharmacol Res 63:341-351.

Chen Q, Zhou Z, Zhang L, Wang Y, Zhang YW, Zhong M, Xu SC, Chen CH, Li L, Yu ZP (2012) Tau protein is involved in morphological plasticity in hippocampal neurons in response to BDNF. Neurochem Int 60:233-242.

Dayanandan R, Van Slegtenhorst M, Mack TG, Ko L, Yen SH, Leroy K, Brion JP, Anderton BH, Hutton M, Lovestone S (1999) Mutations in tau reduce its microtubule binding properties in intact cells and affect its phosphorylation. FEBS Lett 446:228-232.

Dehmelt L, Halpain S (2005) The MAP2/Tau family of microtubuleassociated proteins. Genome Biol 6:204.

Derry WB, Wilson L, Jordan MA (1995) Substoichiometric binding of taxol suppresses microtubule dynamics. Biochemistry 34:2203-2211.

Drechsel DN, Hyman AA, Cobb MH, Kirschner MW (1992) Modulation of the dynamic instability of tubulin assembly by the microtubule-associated protein tau. Mol Biol Cell 3:1141-1154.

Fanara P, Turner S, Busch R, Killion S, Awada M, Turner H, Mahsut A, Laprade KL, Stark JM, Hellerstein MK (2004) In vivo measurement of microtubule dynamics using stable isotope labeling with heavy water. Effect of taxanes. J Biol Chem 279:49940-49947.

Fanara P, Banerjee J, Hueck RV, Harper MR, Awada M, Turner H, Husted KH, Brandt R, Hellerstein MK (2007) Stabilization of hyperdynamic microtubules is neuroprotective in amyotrophic lateral sclerosis. J Biol Chem 282:23465-23472.

Fanara P, Husted KH, Selle K, Wong PY, Banerjee J, Brandt R, Hellerstein MK (2010) Changes in microtubule turnover accompany synaptic plasticity and memory formation in response to contextual fear conditioning in mice. Neuroscience 168:167-178.
Feinstein SC, Wilson L (2005) Inability of tau to properly regulate neuronal microtubule dynamics: a loss-of-function mechanism by which tau might mediate neuronal cell death. Biochim Biophys Acta 1739:268-279.

Gardiner J, Overall R, Marc J (2011) The microtubule cytoskeleton acts as a key downstream effector of neurotransmitter signaling. Synapse 65:249-256.

Giannakakou P, Nakano M, Nicolaou KC, O’Brate A, Yu J, Blagosklonny MV, Greber UF, Fojo T (2002) Enhanced microtubule-dependent trafficking and $\mathrm{p} 53$ nuclear accumulation by suppression of microtubule dynamics. Proc Natl Acad Sci U S A 99:10855-10860.

Gozes I, Stewart A, Morimoto B, Fox A, Sutherland K, Schmeche D (2009) Addressing Alzheimer's disease tangles: from NAP to AL-108. Curr Alzheimer Res 6:455-460.

Hellal F, Hurtado A, Ruschel J, Flynn KC, Laskowski CJ, Umlauf M, Kapitein LC, Strikis D, Lemmon V, Bixby J, Hoogenraad CC, Bradke F (2011) Microtubule stabilization reduces scarring and causes axon regeneration after spinal cord injury. Science 331:928-931.

Hoogenraad CC, Akhmanova A (2010) Dendritic spine plasticity: new regulatory roles of dynamic microtubules. Neuroscientist 16:650-661.

Hoover BR, Reed MN, Su J, Penrod RD, Kotilinek LA, Grant MK, Pitstick R, Carlson GA, Lanier LM, Yuan LL, Ashe KH, Liao D (2010) Tau mislocalization to dendritic spines mediates synaptic dysfunction independently of neurodegeneration. Neuron 68:1067-1081.

Ittner A, Ke YD, Eersel J, Gladbach A, Götz J, Ittner LM (2011) Brief update on different roles of tau in neurodegeneration. IUBMB Life 63:495-502.

Ittner LM, Gotz J (2011) Amyloid-beta and tau-a toxic pas de deux in Alzheimer's disease. Nat Rev Neurosci 12:65-72.

Jiang K, Akhmanova A (2011) Microtubule tip-interacting proteins: a view from both ends. Curr Opin Cell Biol 23:94-101.

Job D, Rauch CT, Fischer EH, Margolis RL (1983) Regulation of microtubule cold stability by calmodulin-dependent and -independent phosphorylation. Proc Natl Acad Sci U S A 80:3894-3898.

Kolman A (2004) Epothilone D (Kosan/Roche). Curr Opin Investig Drugs 5:657-667.

Lace GL, Wharton SB, Ince PG (2007) A brief history of tau: the evolving view of the microtubule-associated protein tau in neurodegenerative diseases. Clin Neuropathol 26:43-58.

Lee S, Sato Y, Nixon RA (2011) Lysosomal proteolysis inhibition selectively disrupts axonal transport of degradative organelles and causes an Alzheimer's-like axonal dystrophy. J Neurosci 31:7817-7830.

Lis G, Wassenaar LI, Hendry MJ (2008) High-precision laser spectroscopy $\mathrm{D} / \mathrm{H}$ and $18 \mathrm{O} / 16 \mathrm{O}$ measurements of microliter natural water samples. Anal Chem 80:287-293.

Lu Q, Wood JG (1993) Functional studies of Alzheimer's disease tau protein. J Neurosci 13:508-515.

Manna T, Thrower DA, Honnappa S, Steinmetz MO, Wilson L (2009) Regulation of microtubule dynamic instability in vitro by differentially phosphorylated stathmin. J Biol Chem 284:15640-15649.

Michaelis ML, Chen Y, Hill S, Reiff E, Georg G, Rice A, Audus K (2002) Amyloid peptide toxicity and microtubule-stabilizing drugs. J Mol Neurosci 19:101-105.

Michaelis ML, Seyb KI, Ansar S (2005) Cytoskeletal integrity as a drug target. Curr Alzheimer Res 2:227-229.

Mocanu MM, Nissen A, Eckermann K, Khlistunova I, Biernat J, Drexler D, Petrova O, Schönig K, Bujard H, Mandelkow E, Zhou L, Rune G, Mandelkow EM (2008) The potential for $\beta$-structure in the repeat domain of tau protein determines aggregation, synaptic decay, neuronal loss, and coassembly with endogenous tau in inducible mouse models of tauopathy. J Neurosci 28:737-748

Montoro RJ, Díaz-Nido J, Avila J, López-Barneo J (1993) N-methyl-Daspartate stimulates the dephosphorylation of the microtubule-associated protein 2 and potentiates excitatory synaptic pathways in the rat hippocampus. Neuroscience 54:859-871.

Morris M, Maeda S, Vossel K, Mucke L (2011) The many faces of tau. Neuron $70: 410-426$

Oddo S, Caccamo A, Shepherd JD, Murphy MP, Golde TE, Kayed R, Metherate R, Mattson MP, Akbari Y, LaFerla FM (2003) Triple-transgenic model of Alzheimer's disease with plaques and tangles: intracellular Abeta and synaptic dysfunction. Neuron 39:409-421.

Planel E, Krishnamurthy P, Miyasaka T, Liu L, Herman M, Kumar A, Bretteville A, Figueroa HY, Yu WH, Whittington RA, Davies P, Takashima A, Nixon RA, Duff KE (2008) Anesthesia-induced hyperphosphorylation 
detaches 3-repeat tau from microtubules without affecting their stability in vivo. J Neurosci 28:12798-12807.

Qiang L, Yu W, Andreadis A, Luo M, Baas PW (2006) Tau protects microtubules in the axon from severing by katanin. J Neurosci 26:3120-3129.

Ramsden M, Kotilinek L, Forster C, Paulson J, McGowan E, SantaCruz K, Guimaraes A, Yue M, Lewis J, Carlson G, Hutton M, Ashe KH (2005) Age-dependent neurofibrillary tangle formation, neuron loss, and memory impairment in a mouse model of human tauopathy (P301L). J Neurosci 25:10637-10647.

Santacruz K, Lewis J, Spires T, Paulson J, Kotilinek L, Ingelsson M, Guimaraes A, DeTure M, Ramsden M, McGowan E, Forster C, Yue M, Orne J, Janus C, Mariash A, Kuskowski M, Hyman B, Hutton M, Ashe KH (2005) Tau suppression in a neurodegenerative mouse model improves memory function. Science 309:476-481.

Sengottuvel V, Leibinger M, Pfreimer M, Andreadaki A, Fischer D (2011) Taxol facilitates axon regeneration in the mature CNS. J Neurosci 31:2688-2699.

Shahpasand K, Uemura I, Saito T, Asano T, Hata K, Shibata K, Toyoshima Y, Hasegawa M, Hisanaga S (2012) Regulation of mitochondrial transport and inter-microtubule spacing by tau phosphorylation at the sites hyperphosphorylated in Alzheimer's disease. J Neurosci 32:2430-2441.

Shemesh OA, Spira ME (2010) Hallmark cellular pathology of Alzheimer's disease induced by mutant human tau expression in cultured Aplysia neurons. Acta neuropathologica 120:209-222.

Shemesh OA, Spira ME (2011) Rescue of neurons from undergoing hallmark tau-induced Alzheimer's disease cell pathologies by the antimitotic drug paclitaxel. Neurobiol Dis 43:163-175.

Shemesh OA, Erez H, Ginzburg I, Spira ME (2008) Tau-induced traffic jams reflect organelles accumulation at points of microtubule polar mismatching. Traffic 9:458-471.

Silva DF, Esteves AR, Arduino DM, Oliveira CR, Cardoso SM (2011) Amyloid-beta-induced mitochondrial dysfunction impairs the au- tophagic lysosomal pathway in a tubulin dependent pathway. J Alzheimers Dis 26:565-581.

Spires TL, Orne JD, SantaCruz K, Pitstick R, Carlson GA, Ashe KH, Hyman BT (2006) Region-specific dissociation of neuronal loss and neurofibrillary pathology in a mouse model of tauopathy. Am J Pathol 168:1598-1607.

Vossel KA, Zhang K, Brodbeck J, Daub AC, Sharma P, Finkbeiner S, Cui B, Mucke L (2010) Tau reduction prevents Abeta-induced defects in axonal transport. Science 330:198.

Weissmann C, Reyher HJ, Gauthier A, Steinhoff HJ, Junge W, Brandt R (2009) Microtubule binding and trapping at the tip of neurites regulate tau motion in living neurons. Traffic 10:1655-1668.

Yenjerla M, LaPointe NE, Lopus M, Cox C, Jordan MA, Feinstein SC, Wilson L (2010) The neuroprotective peptide NAP does not directly affect polymerization or dynamics of reconstituted neural microtubules. J Alzheimers Dis 19:1377-1386.

Zempel H, Mandelkow EM (2011) Linking amyloid- $\beta$ and tau: amyloid- $\beta$ induced synaptic dysfunction via local wreckage of the neuronal cytoskeleton. Neurodegener Dis. Advance online publication. Retrieved April 11, 2012. doi:10.1159/000332816.

Zhang B, Maiti A, Shively S, Lakhani F, McDonald-Jones G, Bruce J, Lee EB, Xie SX, Joyce S, Li C, Toleikis PM, Lee VM, Trojanowski JQ (2005) Microtubule-binding drugs offset tau sequestration by stabilizing microtubules and reversing fast axonal transport deficits in a tauopathy model. Proc Natl Acad Sci U S A 102:227-231.

Zhang B, Carroll J, Trojanowski JQ, Yao Y, Iba M, Potuzak JS, Hogan AM, Xie SX, Ballatore C, Smith AB 3rd, Lee VM, Brunden KR (2012) The microtubule-stabilizing agent, epothilone $\mathrm{D}$, reduces axonal dysfunction, neurotoxicity, cognitive deficits, and Alzheimer-like pathology in an interventional study with aged tau transgenic mice. J Neurosci 32:36013611. 